Article

\title{
Existence of Solutions for Anti-Periodic Fractional Differential Inclusions Involving $\psi$-Riesz-Caputo Fractional Derivative
}

\author{
Dandan Yang *(D) and Chuanzhi Bai \\ School of Mathematical Science, Huaiyin Normal University, Huaian 223300, Jiangsu, China \\ * Correspondence: ydd@hytc.edu.cn
}

Received: 9 June 2019; Accepted: 12 July 2019; Published: 15 July 2019

\begin{abstract}
In this paper, we investigate the existence of solutions for a class of anti-periodic fractional differential inclusions with $\psi$-Riesz-Caputo fractional derivative. A new definition of $\psi$-Riesz-Caputo fractional derivative of order $\alpha$ is proposed. By means of Contractive map theorem and nonlinear alternative for Kakutani maps, sufficient conditions for the existence of solutions to the fractional differential inclusions are given. We present two examples to illustrate our main results.
\end{abstract}

Keywords: fractional differential inclusions; $\psi$-Riesz-Caputo derivative; existence of solutions; fixed point theorem; anti-periodic boundary value problems

\section{Introduction}

Fractional order models, providing excellent description of memory and hereditary processes, are more adequate than integer order ones. Some recent contributions to fractional differential equations and inclusions have been carried out, see the monographs [1-8], and the references cited therein. The study of fractional differential equations or inclusions with anti-periodic boundary problems, which are applied in different fields, such as physics, chemical engineering, economics, populations dynamics and so on, have recently received considerable attention, see the references $([9,10])$ and papers cited therein. There are several definitions of fractional differential derivatives and integrals, such like Caputo type, Rimann-Liouville type, Hadamard type and Erdelyi-Kober type and so on. In order to develop the fractional calculus, some different and special form of differential operators are chosen, for example, see [11-15] and the references therein. The $\alpha$ order $\psi$-Caputo fractional derivative was first introduced by Almeida in [3]. Some properties, like semigroup law, Taylor's Theorem, Fermat's Thorem, etc., were presented. This newly defined fractional derivative could model more accurately the process using differential kernels for the fractional operator. In 2018, Samet and Aydi in [16] considered the following fractional differential equation with anti-periodic boundary conditions:

$$
\left\{\begin{array}{l}
{ }^{c} D^{\alpha, \psi} u(x)+f(x, u(x))=0, \quad a<x<b, \\
u(a)+u(b)=0, u^{\prime}(a)+u^{\prime}(b)=0
\end{array}\right.
$$

where $(a, b) \in R^{2}, a<b, 1<\alpha<2, \psi \in C^{2}([a, b]), \psi^{\prime}(x)>0, x \in[a, b]{ }^{c} D^{\alpha, \psi}$ is the $\psi$-Caputo fractional derivative of order $\alpha$, and $f:[a, b] \times R \rightarrow R$ is a given function. A Lyapunov-type inequality is established for problem (1). The authors also give some examples to illustrate the applications of their main results.

Very recently, Chen et al. in [10] studied the following anti-periodic boundary problem involving the Riesz-Caputo derivative 


$$
\left\{\begin{array}{l}
{ }_{0}^{R C} D_{T}^{\gamma} y(\tau)=g(\tau, y(\tau)), \quad \tau \in[0, T], 1<\gamma \leq 2, \\
y(0)=-y(T), \quad y^{\prime}(0)=-y^{\prime}(T),
\end{array}\right.
$$

where ${ }_{0}^{R C} D_{T}^{\gamma}$ is a Riesz-Caputo derivative, which can reflect both the past and the future nonlocal memory effects and $g:[0, T] \times R \rightarrow R$ is a continuous function with respect to $\tau$ and $y$. Some existence results of solutions are given based on the Lipschitz condition, the growth condition and the comparison condition. Most of the present work are concerned with fractional differential equations or inclusions involving Riemann-Liouville or Caputo fractional derivative, merely reflecting the past or future memory effect. Riesz derivative is a two-sided fractional operator, whose advantage is that it could reflect both the past and the future memory effects. We take anomalous diffusion problem for example. The fractional differential equation with the Riesz derivative is adopted to describe the anomalous diffusion problem, in which the Riesz derivative stands for the nonlocality and the dependence on path of the diffusion concentration. Some applications of Riesz derivative about anomalous diffusion, we refer the reader to $[17,18]$. Another typical example is stocks. According to the price trend of the past and future time, investors would buy or sell a stock at an agreed-on price within a period of time. This process depends on both past state and its development in the future, which is the characteristic of Riesz derivative. There are some other applications of this derivative, and we refer the reader to $[10,19,20]$. In 2009, Ahamad and Otero-Espinar [1] investigated the following fractional inclusions with anti-periodic boundary conditions

$$
\left\{\begin{array}{l}
{ }^{c} D^{q} x(t) \in F(t, x(t)), \quad t \in[0, T], 1<q \leq 2, \\
x(0)=-x(T), \quad x^{\prime}(0)=-x^{\prime}(T),
\end{array}\right.
$$

where ${ }^{c} D^{q} x(t)$ is the standard Caputo derivative of order $q, F:[0, T] \times R \rightarrow \mathcal{P}(R)$ is a multivalued map, $\mathcal{P}(R)$ is the family of all subsets of $R$. Some sufficient conditions for the existence of solutions are given by means of Bohnenblust-Karlin fixed point theorem.

Inspired by the above-mentioned works, in this paper, we are concerned with the following anti-periodic fractional inclusions with $\psi$-Riesz-Caputo derivative:

$$
\left\{\begin{array}{l}
{ }_{a}^{R C} D_{b}^{\alpha, \psi} u(x) \in F(x, u(x)), \quad a<x<b, \\
u(a)+u(b)=0, u^{\prime}(a)+u^{\prime}(b)=0,
\end{array}\right.
$$

where $(a, b) \in R^{2}, a<b, 1<\alpha<2, \psi \in C^{2}([a, b]), \psi^{\prime}(x)>0, x \in[a, b] \cdot{ }_{a}^{R C} D_{b}^{\alpha, \psi}$ is the $\psi$-Riesz-Caputo fractional derivative of order $\alpha$, and $F:[a, b] \times R \rightarrow \mathcal{P}(R)$ is a multivalued map. Sufficient conditions for the existence of solutions are given in view of the fixed point theorems for multi-valued mapping. The aim of this paper is to develop the calculus of fractional derivatives. We shall combine the two definitions of Riesz-Caputo derivative and $\psi$-Caputo fractional derivative. Then we investigate the existence of solutions of anti-periodic inclusions (4). The rest of this paper is organized as follows. We first present some basic definitions of fractional calculus, $\psi$-Caputo derivative, Riesz-Caputo derivative and multi-valued maps, and then a new definition of $\psi$-Riesz-Caputo fractional derivative of order $\alpha$ is given. In Section 3, the main results on the existence of solutions for anti-periodic boundary value problem (4) are provided. We present two examples in order to illustrate our main results in last section. Our results generalize some published known results. There is no literature to research the fractional differential inclusions with $\psi$-Riesz-Caputo fractional derivative. If we take $F(x, u)=\{f(x, u)\}$, where $f:[a, b] \times R \rightarrow R$ is a given continuous function, then the problem (4) corresponds to the single-valued problem (1). If we take $a=0, b=T, \psi(x)=x, F(x, u)=\{g(x, u)\}$, where $g:[0, T] \times R \rightarrow R$ is a given continuous function, then the problem (4) corresponds to the single-valued problem (2). 


\section{Preliminaries}

In this section, we recall some notation, definitions and preliminaries about fractional calculus $[6,7,21], \psi$-Caputo fractional calculus [3-5,22,23], and Riesz or Riesz-Caputo fractional derivative [17-19].

Definition 1 ([6]). The left Caputo fractional derivative order $\alpha(1<\alpha \leq 2)$ of a function $f \in C^{2}([a, b])$ is given by

$$
{ }_{a}^{C} D_{x}^{\alpha} f(x)=\left({ }_{a} I_{x}^{2-\alpha} f^{\prime \prime}\right)(x), a<x<b
$$

that is,

$$
{ }_{a}^{C} D_{x}^{\alpha} f(x)=\frac{1}{\Gamma(2-\alpha)} \int_{a}^{x}(x-t)^{1-\alpha} f^{\prime \prime}(t) d t, a<x<b .
$$

Similarly, the right Caputo fractional integral order $\alpha(1<\alpha \leq 2)$ of a function $f \in C^{2}([a, b])$ is given by

$$
{ }_{x}^{C} D_{b}^{\alpha} f(x)=\frac{1}{\Gamma(2-\alpha)} \int_{x}^{b}(t-x)^{1-\alpha} f^{\prime \prime}(t) d t, a<x<b .
$$

Definition 2 ([6]). The fractional left, right and Riemann-Liouville integrals of order $\beta>0$ are defined as

$$
\begin{aligned}
& { }_{a} I_{\tau}^{\beta} g(\tau)=\frac{1}{\Gamma(\beta)} \int_{a}^{\tau}(\tau-s)^{\beta-1} g(s) d s, \\
& { }_{\tau} I_{b}^{\beta} g(\tau)=\frac{1}{\Gamma(\beta)} \int_{\tau}^{b}(s-\tau)^{\beta-1} g(s) d s \\
& { }_{a} I_{b}^{\beta} g(\tau)=\frac{1}{\Gamma(\beta)} \int_{a}^{b}|s-\tau|^{\beta-1} g(s) d s .
\end{aligned}
$$

Let $\psi \in C^{2}([a, b])$ be a given function such that

$$
\psi^{\prime}(x)>0, a \leq x \leq b
$$

Definition 3 ([3]). The fractional left, right integral of order $\alpha>0$ of a function $f \in C([a, b])$ with respect to $\psi$ are defined by

$$
\begin{aligned}
& \left({ }_{a} I_{x}^{\alpha, \psi}\right) f(x)=\frac{1}{\Gamma(\alpha)} \int_{a}^{x} \psi^{\prime}(t)(\psi(x)-\psi(t))^{\alpha-1} f(t) d t, a \leq x \leq b \\
& \left({ }_{x} I_{b}^{\alpha, \psi}\right) f(x)=\frac{1}{\Gamma(\alpha)} \int_{x}^{b} \psi^{\prime}(t)(\psi(t)-\psi(x))^{\alpha-1} f(t) d t, a \leq x \leq b .
\end{aligned}
$$

Definition 4 ([3]). The left, right $\psi$-Caputo fractional derivative of order $\alpha(1<\alpha \leq 2)$ of a function $f \in C^{2}([a, b])$ are defined as

$$
\begin{aligned}
& { }_{a}^{C} D_{x}^{\alpha, \psi} f(x)=\frac{1}{\Gamma(2-\alpha)} \int_{a}^{x} \psi^{\prime}(t)(\psi(x)-\psi(t))^{1-\alpha}\left(\frac{1}{\psi^{\prime}(t)} \frac{d}{d t}\right)^{2} f(t) d t, a<x<b, \\
& { }_{x}^{C} D_{b}^{\alpha, \psi} f(x)=\frac{1}{\Gamma(2-\alpha)} \int_{x}^{b} \psi^{\prime}(t)(\psi(t)-\psi(x))^{1-\alpha}\left(\frac{1}{\psi^{\prime}(t)} \frac{d}{d t}\right)^{2} f(t) d t, a<x<b .
\end{aligned}
$$

Remark 1. Consider $\psi(x)=x, \psi(x)=\ln x$, the Riemann-Liouville and Hadamard fractional operators are obtained.

Inspired by the above definitions, we shall present a new definition of $\psi$-Riesz-Caputo fractional derivative of order $\alpha$, which is a combination of $\psi$-Caputo fractional derivative and Riesz-Caputo fractional derivative. 
Definition 5. Let $f \in C^{2}([a, b])$. For $x \in[a, b]$, the $\psi$-Riesz-Caputo fractional derivative ${ }_{a}^{R C} D_{b}^{\alpha, \psi} f(x)$ of order $\alpha(1<\alpha \leq 2)$ could be defined by

$$
{ }_{a}^{R C} D_{b}^{\alpha, \psi} f(x)=\frac{1}{2}\left({ }_{a}^{C} D_{x}^{\alpha, \psi}+{ }_{x}^{C} D_{b}^{\alpha, \psi}\right) f(x) .
$$

If we take $\psi(x)=x$, it follows from (7)-(9) that the classic Riesz-Caputo derivative fractional order $\alpha(1<\alpha \leq$ 2) of a function $f \in C^{2}([a, b])$ is given by

$$
{ }_{a}^{R C} D_{b}^{\alpha} f(x)=\frac{1}{2}\left({ }_{a}^{C} D_{x}^{\alpha}+{ }_{x}^{C} D_{b}^{\alpha}\right) f(x),
$$

which is defined as in [19]. For convenience, denote

$$
\begin{aligned}
& P_{c l}(X)=\{Y \in \mathcal{P}(X): Y \quad \text { is closed }\} \\
& P_{b}(X)=\{Y \in \mathcal{P}(X): Y \quad \text { is bounded }\} \\
& P_{c p}(X)=\{Y \in \mathcal{P}(X): Y \quad \text { is compact }\} \\
& P_{c p, c}(X)=\{Y \in \mathcal{P}(X): Y \quad \text { is convex and compact }\} .
\end{aligned}
$$

The following are definitions and properties concerning multi-valued maps [24-28] which will be used in the remainder of this paper.

Definition 6 ([28]). A multivalued map $G: X \rightarrow \mathcal{P}(X)$ :

(a) denote the set $\operatorname{Gr}(G)=\{(x, y) \in X \times Y, y \in G(x)\}$ as the graph of $G$,

$$
t \mapsto d(y, G(t))=\inf \{|y-z|: z \in G(t)\}
$$

is measurable.

(b) if $G: X \rightarrow \mathcal{P}_{c l}(X)$ is called $\gamma$-Lipschitz if and only if there exists $\gamma>0$ such that

$$
H_{d}(N(x), N(y)) \leq \gamma d(x, y) \text {, for each } x, y \in X .
$$

(c) if $G: X \rightarrow \mathcal{P}_{c l}(X)$ is called contraction if and only if it is $\gamma$-Lipschitz with $\gamma<1$.

(d) $G$ is said to be measurable if for every $y \in R$, the function

Definition 7 ([26]). Assume that $F: J \times R \rightarrow \mathcal{P}(R)$ is a multivalued map with nonempty compact values. Denote a multivalued operator $\mathcal{F}: C(J \times R) \rightarrow \mathcal{P}\left(L^{1}(J, R)\right.$ associated with $F$ as

$$
\mathcal{F}(x)=\left\{w \in L^{1}(J, R): w(t) \in F(t, x(t))\right\}
$$

for a.e. $t \in J:=[a, b]$ is a closed interval from $a$ to $b$.

Definition 8 ([26]). Assume that $Y$ is a separable metric space and $N: Y \rightarrow \mathcal{P}\left(L^{1}(J, R)\right)$ is a multivalued operator. If $N$ is lower semi-continuous(l.s.c.) and has nonempty closed and decomposable values, we say $N$ has a property $(B C)$.

Definition 9 ([28]). For each $u \in C(J, R), t \in J=[a, b]$, denote the selection set of $F$ as

$$
S_{F, y}:=\left\{f \in L^{1}(J, R): f(t) \in F(t, u(t)) \text { a.e. } t \in J\right\} .
$$

Definition 10 ([28]). Let $A, B \in \mathcal{P}_{c l}(X$.$) The Pompeiu-Hausdorff distance of A, B$ is defined by

$$
H_{d}(A, B)=\max \left\{\sup _{a \in A} d(a, B), \sup _{b \in B} d(A, b)\right\},
$$


where $d(A, b)=\inf _{a \in A} d(a, b), d(a, B)=\inf _{b \in B} d(a, b)$.

Property 1 ([24]). Let $G$ be a completely continuous multi-valued map with nonempty compact values, then $T$ is u.s.c. $\Longleftrightarrow G$ has a closed graph.

The following lemmas play important roles in the proof of our main results.

Lemma 1 ([28]). (Nonlinear alternative for Kakutani maps ). Assume that $E$ is a Banach space, $C$ is a closed convex subset of $E$, and $U$ is an open subset of $C$ with $0 \in U$. Let $F: \bar{U} \rightarrow \mathcal{P}_{c, c v}(C)$ be a upper semicontinuous compact map. Then either

(i) F has a fixed point in $\bar{U}$, or

(ii) there exist $a u \in \partial U$ and $\lambda \in(0,1)$ satisfying $u \in \lambda F(u)$.

Lemma 2 ([29]). Let $(X, d)$ be a complete metric space. If $N: X \rightarrow \mathcal{P}_{c l}(X)$ is a contraction, then Fix $N \neq \varnothing$.

Lemma 3 ([30]). Let $X$ be a Banach space, and $F: J \times X \rightarrow(P)(X)$ be a $L^{1}$-Carathédory set-valued map with $S_{F} \neq \varnothing$ and let $\Theta: L^{1}(J, X) \rightarrow C(J, X)$ be a linear continuous mapping. Then the set-valued map $\Gamma \circ S_{F}: C(J, X) \rightarrow \mathcal{P}(C(J, X))$ defined by

$$
\left(\Theta \circ S_{F}\right)(u): C(J \times X) \rightarrow \mathcal{P}_{c p, c}(C(J, X)), x \mapsto\left(\Theta \circ S_{F}\right)(u)=\Theta\left(S_{F, u}\right)
$$

is a closed graph operator in $C(J, X) \times C(J, X)$.

Lemma 4 ([20]). Assume that $Y$ is a separable metric space and $N: Y \rightarrow \mathcal{P}\left(L^{1}(J, R)\right)$ is a multivalued operator with the property $(B C)$. Then there exists a continuous single-valued function $g: Y \rightarrow L^{1}(J, R)$ satisfying $g(x) \in N(x)$ for every $x \in Y$, i.e., $N$ has a continuous selection.

From [6], we have

Lemma 5. If $1<\beta \leq 2$ and $g \in C^{2}[a, b]$, then

$$
\begin{aligned}
& { }_{a} I_{\tau a}^{\beta C} D_{\tau}^{\beta} g(\tau)=g(\tau)-g(a)-g^{\prime}(a)(\tau-a), \\
& { }_{\tau} I_{b \tau}^{\beta C} D_{b}^{\beta} g(\tau)=g(\tau)-g(b)+g^{\prime}(b)(b-\tau) .
\end{aligned}
$$

From (10) and Lemma 2.1 in [15], for $u \in C^{2}[a, b]$, and $1<\alpha \leq 2$, we have that

$$
\begin{aligned}
{ }_{a} I_{b a}^{\alpha C} D_{b}^{\alpha} u(\tau) & =\frac{1}{2}\left({ }_{a} I_{\tau a}^{\alpha C} D_{\tau}^{\alpha}+{ }_{\tau} I_{b \tau}^{\alpha C} D_{b}^{\alpha}\right) u(\tau) \\
& =u(\tau)-\frac{1}{2}(u(a)+u(b))-\frac{1}{2} u^{\prime}(a)(\tau-a)+\frac{1}{2} u^{\prime}(b)(b-\tau) .
\end{aligned}
$$

By (11), similar to the proof of Lemma 2.2 in [10], we have the following lemma.

Lemma 6. Assume that $h \in C[a, b]$. A function $u \in C^{2}[a, b]$ given by

$$
\begin{aligned}
u(t)=- & \frac{b-a}{2 \Gamma(\alpha-1)} \int_{a}^{b}(b-s)^{\alpha-2} h(s) d s \\
& +\frac{1}{\Gamma(\alpha)} \int_{a}^{t}(t-s)^{\alpha-1} h(s) d s+\frac{1}{\Gamma(\alpha)} \int_{t}^{b}(s-t)^{\alpha-1} h(s) d s,
\end{aligned}
$$

is a unique solution of the following anti-periodic boundary value problem

$$
\left\{\begin{array}{l}
\left({ }_{a}^{R C} D_{b}^{\alpha} u\right)(t)=h(t), \quad t \in(a, b), 1<\alpha \leq 2, \\
u(a)+u(b)=0, \quad u^{\prime}(a)+u^{\prime}(b)=0 .
\end{array}\right.
$$


As the same argument of Lemma 2.1 in [16], we can easily obtain the following result, which plays a very important role in proving the main results.

Lemma 7. If $f, \psi \in C^{2}([a, b])$, and $\psi^{\prime}(x)>0$ for each $x \in[a, b]$, then

$$
\left({ }_{a}^{R C} D_{x}^{\alpha, \psi} f\right)\left(\psi^{-1}(y)\right)=\left({ }_{\psi(a)}^{R C} D_{\psi(x)}^{\alpha}\left(f \circ \psi^{-1}\right)\right)(y), \psi(a)<y<\psi(b),
$$

and

Moreover, we have

$$
\left({ }_{x}^{R C} D_{b}^{\alpha, \psi} f\right)\left(\psi^{-1}(y)\right)=\left({ }_{\psi(x)}^{R C} D_{\psi(b)}^{\alpha}\left(f \circ \psi^{-1}\right)\right)(y), \psi(a)<y<\psi(b) .
$$

$$
\left({ }_{a}^{R C} D_{b}^{\alpha, \psi} f\right)\left(\psi^{-1}(y)\right)=\left({ }_{\psi(a)}^{R C} D_{\psi(b)}^{\alpha}\left(f \circ \psi^{-1}\right)\right)(y), \psi(a)<y<\psi(b) .
$$

Lemma 8. If $f:[a, b] \times R \rightarrow R, \psi \in C^{2}[a, b]$ with $\psi^{\prime}(x)>0$, and $\psi^{\prime}(a)=\psi^{\prime}(b)$, then the problem

$$
\left\{\begin{array}{l}
{ }_{a}^{R C} D_{b}^{\alpha, \psi} u(x)=f(x, u(x)), \quad a<x<b \\
u(a)+u(b)=0, u^{\prime}(a)+u^{\prime}(b)=0
\end{array}\right.
$$

could be transformed into the following problem

$$
\left\{\begin{array}{l}
R C D_{\psi}^{\alpha} v(y)=f\left(\psi^{-1}(y), v(y)\right), \quad \psi(a)<y<\psi(b), \\
\psi(q(\psi))+v(\psi(b))=0, \quad v^{\prime}(\psi(a))+v^{\prime}(\psi(b))=0 .
\end{array}\right.
$$

A nontrivial solution to (18) is given by $\quad v(y)=-\frac{B-A}{2 \Gamma(\alpha-1)} \int_{A}^{B}(B-s)^{\alpha-2} f\left(\psi^{-1}(s), v(s)\right) d s$

$$
+\frac{1}{\Gamma(\alpha)} \int_{A}^{y}(y-s)^{\alpha-1} f\left(\psi^{-1}(s), v(s)\right) d s+\frac{1}{\Gamma(\alpha)} \int_{y}^{B}(s-y)^{\alpha-1} f\left(\psi^{-1}(s), v(s)\right) d s,
$$

where $A=\psi(a)$ and $B=\psi(b)$.

Proof. We introduce the function $v:[\psi(a), \psi(b)] \rightarrow R$, defined by

$$
v(y)=u\left(\psi^{-1}(y)\right), \psi(a) \leq y \leq \psi(b) .
$$

In virtue of (16), one has

$$
\underset{\psi(a)}{R C} D_{\psi(b)}^{\alpha} v\left(\psi^{-1}(y)\right)={ }_{a}^{R C} D_{b}^{\alpha, \psi} u(x), \quad \psi(a)<y<\psi(b) .
$$

By a chain rule, we have

$$
v^{\prime}(y)=\frac{1}{\psi^{\prime}\left(\psi^{-1}(y)\right)} u^{\prime}\left(\psi^{-1}(y)\right), \psi(a) \leq y \leq \psi(b) .
$$

Thus, we have

$$
v^{\prime}(\psi(a))=\frac{1}{\psi^{\prime}(a)} u^{\prime}(a) \text {, and } v^{\prime}(\psi(b))=\frac{1}{\psi^{\prime}(b)} u^{\prime}(b) .
$$

From boundary condition (17) and condition $\psi^{\prime}(a)=\psi^{\prime}(b)$, we have that

$$
v(\psi(a))+v(\psi(b))=0, v^{\prime}(\psi(a))+v^{\prime}(\psi(b))=0 .
$$

Therefore, the problem (17) could be transformed into problem (18). By virtue of Lemma 6, we obtain $v \in C^{2}[A, B]$ is a nontrivial solution to (18).

From Lemma 8, we can easily know that 


$$
\begin{aligned}
& u(x)=-\frac{\psi(b)-\psi(a)}{2 \Gamma(\alpha-1)} \int_{a}^{b}(\psi(b)-\psi(t))^{\alpha-2} \psi^{\prime}(t) f(t, u(t)) d t \\
& +\frac{1}{\Gamma(\alpha)} \int_{a}^{x}(\psi(x)-\psi(t))^{\alpha-1} \psi^{\prime}(t) f(t, u(t)) d t+\frac{1}{\Gamma(\alpha)} \int_{x}^{b}(\psi(t)-\psi(x))^{\alpha-1} \psi^{\prime}(t) f(t, u(t)) d t
\end{aligned}
$$

is a unique solution of problem (17).

\section{Main Results}

We pose the following hypotheses:

$\left(H_{1}\right) F:[a, b] \times R \rightarrow \mathcal{P}(R)$ is Carathéodory and it has nonempty compact and convex values;

$\left(H_{2}\right)$ there exist a continuous nondecreasing function $q:[0, \infty) \rightarrow[0, \infty)$ and a function $p \in C\left([a, b], R^{+}\right)$satisfying

$$
\|F(t, u)\|:=\sup \{|f|: f \in F(t, u)\} \leq p(t) q(\|u\|), \text { for each }(t, x) \in[a, b] \times R .
$$

$\left(H_{3}\right) 1<\alpha<2, \psi \in C^{2}([a, b]), \psi^{\prime}(x)>0, x \in[a, b]$.

$\left(H_{4}\right) \psi^{\prime}(a)=\psi^{\prime}(b)$.

$\left(H_{5}\right) F:[a, b] \times R \rightarrow \mathcal{P}_{c p}(R)$ is such that, for every $u \in R, F(\cdot, u)$ is measurable.

$\left(H_{6}\right)$ There exists $\left.m \in L^{1}([a, b)], R^{+}\right)$for almost all $t \in[a, b]$, such that

$$
d_{H}(F(t, u), F(t, \bar{u})) \leq m(t)|u-\bar{u}|, \quad \forall u, \bar{u} \in R
$$

with $d(0, F(t, 0)) \leq m(t)$ for almost all $t \in[a, b]$.

$\left(H_{7}\right) F:[a, b] \times R \rightarrow \mathcal{P}(R)$ is a nonempty compact-valued multivalued map such that

(a) $\quad(x, u) \mapsto F(x, u)$ is $\mathcal{L} \otimes \mathcal{B}$ is measurable.

(b) $u \mapsto F(x, u)$ is lower semicontinuous for each $x \in[a, b]$,

Now we are in the position to state our main results. The first theorem is dealing with the Carathéodory case.

Theorem 1. Assume that $\left(H_{1}\right)-\left(H_{4}\right)$ hold. Moreover, if there exists a constant $M>0$, such that

$$
\begin{gathered}
M\left[q ( M ) \left(\frac{\psi(b)-\psi(a)}{2 \Gamma(\alpha-1)} \int_{a}^{b}(\psi(b)-\psi(s))^{\alpha-2} p(s) \psi^{\prime}(s) d s\right.\right. \\
\left.\left.+\frac{2}{\Gamma(\alpha)}(\psi(b)-\psi(a))^{\alpha-1} \int_{a}^{b} p(s) \psi^{\prime}(s) d s\right)\right]^{-1}>1
\end{gathered}
$$

Then (4) has at least one solution on $[a, b]$.

Proof. The operator $T: C([a, b], R) \rightarrow \mathcal{P}(C[A, B], R)$ is defined as follows:

$$
\begin{aligned}
T(u) & =\left\{h \in C([a, b], R): h(t)=-\frac{\psi(b)-\psi(a)}{2 \Gamma(\alpha-1)} \int_{a}^{b}(\psi(b)-\psi(s))^{\alpha-2} \psi^{\prime}(s) f(s) d s\right. \\
& \left.+\frac{1}{\Gamma(\alpha)} \int_{a}^{t}(\psi(t)-\psi(s))^{\alpha-1} \psi^{\prime}(s) f(s) d s+\frac{1}{\Gamma(\alpha)} \int_{t}^{b}(\psi(s)-\psi(t))^{\alpha-1} \psi^{\prime}(s) f(s) d s, \quad f \in S_{F, u}\right\}
\end{aligned}
$$

We divide the proof into 5 parts, which shows that $T$ satisfies all the conditions of Lemma 1.

Part (i). $T$ maps the bounded sets into bounded sets of $C([a, b], R)$. Set $B_{r}=\{v \in C([a, b], R)$ : $\|v\| \leq r, \quad r>0\}$, which is a bounded ball in $C([a, b], R)$, then for $h \in T(u), u \in B_{r}$, there exists $f \in S_{F, u}$ such that

$$
h(t)=-\frac{\psi(b)-\psi(a)}{2 \Gamma(\alpha-1)} \int_{a}^{b}(\psi(b)-\psi(s))^{\alpha-2} \psi^{\prime}(s) f(s) d s
$$




$$
+\frac{1}{\Gamma(\alpha)} \int_{a}^{t}(\psi(t)-\psi(s))^{\alpha-1} \psi^{\prime}(s) f(s) d s+\frac{1}{\Gamma(\alpha)} \int_{t}^{b}(\psi(s)-\psi(t))^{\alpha-1} \psi^{\prime}(s) f(s) d s .
$$

Then

$$
\begin{aligned}
|h(t)| \leq & \frac{\psi(b)-\psi(a)}{2 \Gamma(\alpha-1)} \int_{a}^{b}(\psi(b)-\psi(s))^{\alpha-2} \psi^{\prime}(s)|f(s)| d s \\
& +\frac{1}{\Gamma(\alpha)} \int_{a}^{t}(\psi(t)-\psi(s))^{\alpha-1} \psi^{\prime}(s)|f(s)| d s+\frac{1}{\Gamma(\alpha)} \int_{t}^{b}(\psi(s)-\psi(t))^{\alpha-1} \psi^{\prime}(s)|f(s)| d s . \\
\leq & q(\|u\|)\left[\frac{\psi(b)-\psi(a)}{2 \Gamma(\alpha-1)} \int_{a}^{b}(\psi(b)-\psi(s))^{\alpha-2} \psi^{\prime}(s) p(s) d s\right. \\
& \left.+\frac{1}{\Gamma(\alpha)} \int_{a}^{t}(\psi(t)-\psi(s))^{\alpha-1} \psi^{\prime}(s) p(s) d s+\frac{1}{\Gamma(\alpha)} \int_{t}^{b}(\psi(s)-\psi(t))^{\alpha-1} \psi^{\prime}(s) p(s) d s\right] \\
\leq & q(r)\left(\frac{\psi(b)-\psi(a)}{2 \Gamma(\alpha-1)} \int_{a}^{b}(\psi(b)-\psi(s))^{\alpha-2} \psi^{\prime}(s) p(s) d s\right. \\
+ & \left.\frac{2}{\Gamma(\alpha)}(\psi(b)-\psi(a))^{\alpha-1} \int_{a}^{b} \psi^{\prime}(s) p(s) d s\right) .
\end{aligned}
$$

Part (ii). $T$ maps bounded set into equicontinuous sets. Let $u \in B_{r}, t_{1}, t_{2} \in[a, b], t_{1}<t_{2}$, where $B_{r}$ is a bounded set in $C([a, b], R)$, for $u \in T(u)$, we have

$$
\begin{aligned}
\left|h\left(t_{2}\right)-h\left(t_{1}\right)\right| \leq & \frac{1}{\Gamma(\alpha)} \int_{a}^{t_{1}}\left[\left(\psi\left(t_{2}\right)-\psi(s)\right)^{\alpha-1}-\left(\psi\left(t_{1}\right)-\psi(s)\right)^{\alpha-1}\right] \psi^{\prime}(s)|f(s)| d s \\
& +\frac{1}{\Gamma(\alpha)} \int_{t_{1}}^{t_{2}}\left[\left(\psi\left(t_{2}\right)-\psi(s)\right)^{\alpha-1}-\left(\psi\left(t_{1}\right)-\psi(s)\right)^{\alpha-1}\right] \psi^{\prime}(s)|f(s)| d s \\
& +\frac{1}{\Gamma(\alpha)} \int_{t_{2}}^{b}\left[\left(\psi\left(t_{2}\right)-\psi(s)\right)^{\alpha-1}-\left(\psi\left(t_{1}\right)-\psi(s)\right)^{\alpha-1}\right] \psi^{\prime}(s)|f(s)| d s \\
\leq & \frac{q(r)}{\Gamma(\alpha)} \int_{a}^{t_{1}}\left[\left(\psi\left(t_{2}\right)-\psi(s)\right)^{\alpha-1}-\left(\psi\left(t_{1}\right)-\psi(s)\right)^{\alpha-1}\right] \psi^{\prime}(s) p(s) d s \\
& +\frac{q(r)}{\Gamma(\alpha)} \int_{t_{1}}^{t_{2}}\left[\left(\psi\left(t_{2}\right)-\psi(s)\right)^{\alpha-1}-\left(\psi\left(t_{1}\right)-\psi(s)\right)^{\alpha-1}\right] \psi^{\prime}(s) p(s) d s \\
& +\frac{q(r)}{\Gamma(\alpha)} \int_{t_{2}}^{b}\left[\left(\psi\left(t_{2}\right)-\psi(s)\right)^{\alpha-1}-\left(\psi\left(t_{1}\right)-\psi(s)\right)^{\alpha-1}\right] \psi^{\prime}(s) p(s) d s
\end{aligned}
$$

independent of $u \in B_{r}$ as $t_{1} \rightarrow t_{2}$, the right side hand of above inequality tends to 0 . According to the Ascoli-Arzelá Theorem, $T$ is completely continuous.

Part (iii). $T$ has a closed graph. Set $u_{n} \rightarrow u_{*}, h_{n} \in T\left(u_{n}\right)$ and $h_{n} \rightarrow h_{*}$. Then, we shall show that $h_{*} \in T\left(u_{*}\right)$. For $h_{n} \in T\left(u_{n}\right)$, there exist $f_{n} \in S_{F, u_{n}}$ such that

$$
\begin{gathered}
h_{n}(t)=-\frac{\psi(b)-\psi(a)}{2 \Gamma(\alpha-1)} \int_{a}^{b}(\psi(b)-\psi(s))^{\alpha-2} \psi^{\prime}(s) f_{n}(s) d s \\
+\frac{1}{\Gamma(\alpha)} \int_{a}^{t}(\psi(t)-\psi(s))^{\alpha-1} \psi^{\prime}(s) f_{n}(s) d s+\frac{1}{\Gamma(\alpha)} \int_{t}^{b}(\psi(s)-\psi(t))^{\alpha-1} \psi^{\prime}(s) f_{n}(s) d s .
\end{gathered}
$$

Hence, it suffices to show that there exists $f_{*} \in S_{F, u_{*}}$, such that for each $t \in[a, b]$,

$$
h_{*}(t)=-\frac{\psi(b)-\psi(a)}{2 \Gamma(\alpha-1)} \int_{a}^{b}(\psi(b)-\psi(s))^{\alpha-2} \psi^{\prime}(s) f_{*}(s) d s
$$




$$
+\frac{1}{\Gamma(\alpha)} \int_{a}^{t}(\psi(t)-\psi(s))^{\alpha-1} \psi^{\prime}(s) f_{*}(s) d s+\frac{1}{\Gamma(\alpha)} \int_{t}^{b}(\psi(s)-\psi(t))^{\alpha-1} \psi^{\prime}(s) f_{*}(s) d s .
$$

Define the continuous linear the operator $\Phi: L^{1}([a, b], R) \rightarrow C([a, b], R)$ :

$$
\begin{aligned}
f & \mapsto \Phi(f)(t)=-\frac{\psi(b)-\psi(a)}{2 \Gamma(\alpha-1)} \int_{a}^{b}(\psi(b)-\psi(s))^{\alpha-2} \psi^{\prime}(s) f(s) d s \\
& +\frac{1}{\Gamma(\alpha)} \int_{a}^{t}(\psi(t)-\psi(s))^{\alpha-1} \psi^{\prime}(s) f(s) d s+\frac{1}{\Gamma(\alpha)} \int_{t}^{b}(\psi(s)-\psi(t))^{\alpha-1} \psi^{\prime}(s) f(s) d s .
\end{aligned}
$$

We have $\left\|h_{n}-h\right\| \rightarrow 0$, as $n \rightarrow \infty$. Thus, in light of Lemma 3, $\Phi \circ S_{F}$ is a closed graph operator. Furthermore, we have $h_{n}(t) \in \Phi\left(S_{F, u_{n}}\right)$. By $u_{n} \rightarrow u_{*}$, we obtain

$$
\begin{aligned}
& h_{*}(t)=-\frac{\psi(b)-\psi(a)}{2 \Gamma(\alpha-1)} \int_{a}^{b}(\psi(b)-\psi(s))^{\alpha-2} \psi^{\prime}(s) f_{*}(s) d s \\
& +\frac{1}{\Gamma(\alpha)} \int_{a}^{t}(\psi(t)-\psi(s))^{\alpha-1} \psi^{\prime}(s) f_{*}(s) d s+\frac{1}{\Gamma(\alpha)} \int_{t}^{b}(\psi(s)-\psi(t))^{\alpha-1} \psi^{\prime}(s) f_{*}(s) d s,
\end{aligned}
$$

for some $f_{*} \in S_{F, u_{*}}$.

Part (iv). $T$ is convex for each $x \in C([a, b], R)$. Since $S_{F, u}$ is convex, it is obviously true.

Part (v). We show that there exists a open set $U \subset C([a, b], R)$, with $u \notin T(u)$ for any $\eta \in(0,1)$ and all $u \in \partial U$. Let $\eta \in(0,1), u \in \eta T(u)$. Then for $t \in[a, b]$, there exists $f \in S_{F, u}$ such that

$$
\begin{aligned}
& h(t)=-\frac{\psi(b)-\psi(a)}{2 \Gamma(\alpha-1)} \int_{a}^{b}(\psi(b)-\psi(s))^{\alpha-2} \psi^{\prime}(s) f(s) d s \\
& +\frac{1}{\Gamma(\alpha)} \int_{a}^{t}(\psi(t)-\psi(s))^{\alpha-1} \psi^{\prime}(s) f(s) d s+\frac{1}{\Gamma(\alpha)} \int_{t}^{b}(\psi(s)-\psi(t))^{\alpha-1} \psi^{\prime}(s) f(s) d s .
\end{aligned}
$$

A similar discussion as in part (i), we have

$$
\begin{aligned}
\|h\| \leq & q(\|u\|)\left(\frac{\psi(b)-\psi(a)}{2 \Gamma(\alpha-1)} \int_{a}^{b}(\psi(b)-\psi(s))^{\alpha-2} \psi^{\prime}(s) p(s) d s\right. \\
& \left.+\frac{2}{\Gamma(\alpha)}(\psi(b)-\psi(a))^{\alpha-1} \int_{a}^{b} \psi^{\prime}(s) p(s) d s\right) .
\end{aligned}
$$

Consequently, we have

$\frac{\|u\|}{q(\|u\|)\left(\frac{\psi(b)-\psi(a)}{2 \Gamma(\alpha-1)} \int_{a}^{b}(\psi(b)-\psi(s))^{\alpha-2} \psi^{\prime}(s) p(s) d s+\frac{2}{\Gamma(\alpha)}(\psi(b)-\psi(a))^{\alpha-1} \int_{a}^{b} \psi^{\prime}(s) p(s) d s\right)} \leq 1$.

By (23), there exists $M$ such that $\|u\| \neq M$. Let

$$
U=\{x \in C([a, b], R):\|u\|<M\} .
$$

It is clear that the operator $T: \bar{U} \rightarrow \mathcal{P}(C([a, b], R))$ is upper semicontinuous and completely continuous. If we choose $U$ properly, for some $\eta \in(0,1)$, there is no $u \in \partial U$ such that $u \in \eta T(u)$. Thus, by means of Lemma 1 , we can get the conclusion that thereexists a fixed point $u \in \bar{U}$, that is, it is a solution of problem (4). We complete the proof.

We shall give the second theorem which is concerned with the Lipschitz case.

Theorem 2. Suppose that the conditions $\left(H_{3}\right)-\left(H_{6}\right)$ are satisfied. Moreover, if

$$
\gamma:=\frac{\psi(b)-\psi(a)}{2 \Gamma(\alpha-1)} \int_{a}^{b}(\psi(b)-\psi(s))^{\alpha-2} \psi^{\prime}(s) m(s) d s+\frac{2}{\Gamma(\alpha)}(\psi(b)-\psi(a))^{\alpha-1} \int_{a}^{b} \psi^{\prime}(s) m(s) d s<1
$$


then problem (4) has at least a solution on $[a, b]$.

Proof. By (22), we define the operator $T: C([a, b], R) \rightarrow \mathcal{P}(C[a, b], R)$ as follows:

$$
\begin{aligned}
& T(u)=\left\{h \in C([a, b], R): h(t)=-\frac{\psi(b)-\psi(a)}{2 \Gamma(\alpha-1)} \int_{a}^{b}(\psi(b)-\psi(s))^{\alpha-2} \psi^{\prime}(s) g(s) d s\right. \\
&+\frac{1}{\Gamma(\alpha)} \int_{a}^{t}(\psi(t)-\psi(s))^{\alpha-1} \psi^{\prime}(s) g(s) d s \\
&\left.+\frac{1}{\Gamma(\alpha)} \int_{t}^{b}(\psi(s)-\psi(t))^{\alpha-1} \psi^{\prime}(s) g(s) d s, \quad g \in S_{F, u}\right\} .
\end{aligned}
$$

Obviously, the fixed point of $T$ is the solution of (4). Our aim is to prove that the operator $T$ satisfies all the conditions in Lemma 2. The proof will be given in two claims.

Claim 1. For each $h \in C([a, b], \mathbb{R})$ the operator $T$ is closed. Let $\left\{h_{n}\right\}_{n \geq 0} \in T(u)$ be such that $h_{n} \rightarrow h(n \rightarrow \infty)$ in $C([a, b], R)$. Then $h \in C([a, b], \mathbb{R})$, and there exists $v_{n} \in S_{F, u}$ such that for each $t \in[a, b]$

$$
\begin{aligned}
& h_{n}(t)=-\frac{\psi(b)-\psi(a)}{2 \Gamma(\alpha-1)} \int_{a}^{b}(\psi(b)-\psi(s))^{\alpha-2} \psi^{\prime}(s) v_{n}(s) d s \\
& +\frac{1}{\Gamma(\alpha)} \int_{a}^{t}(\psi(t)-\psi(s))^{\alpha-1} \psi^{\prime}(s) v_{n}(s) d s+\frac{1}{\Gamma(\alpha)} \int_{t}^{b}(\psi(s)-\psi(t))^{\alpha-1} \psi^{\prime}(s) v_{n}(s) d s .
\end{aligned}
$$

For $F$ has compact values, we get a subsequence $v_{n}$ which converges to $v \in L^{1}([a, b], R)$. Thus, $v \in S_{F, u}$, and for each $t \in[a, b]$, one has

$$
\begin{aligned}
h_{n}(t) & \rightarrow h(t)=-\frac{\psi(b)-\psi(a)}{2 \Gamma(\alpha-1)} \int_{a}^{b}(\psi(b)-\psi(s))^{\alpha-2} \psi^{\prime}(s) v(s) d s \\
& +\frac{1}{\Gamma(\alpha)} \int_{a}^{t}(\psi(t)-\psi(s))^{\alpha-1} \psi^{\prime}(s) v(s) d s+\frac{1}{\Gamma(\alpha)} \int_{t}^{b}(\psi(s)-\psi(t))^{\alpha-1} \psi^{\prime}(s) v(s) d s .
\end{aligned}
$$

Therefore, $h \in T(u)$.

Claim 2. We shall show that there exists $\gamma<1$ such that

$$
H_{d}(F(t, u), F(t, \bar{u})) \leq \gamma\|u-\bar{u}\| .
$$

Let $u, \bar{u} \in C([a, b], R)$ and $h_{1} \in T(u)$. There exists $v_{1}(t) \in F(t, u(t))$ such that for each $t \in[a, b]$,

$$
\begin{gathered}
h_{1}(t)=-\frac{\psi(b)-\psi(a)}{2 \Gamma(\alpha-1)} \int_{a}^{b}(\psi(b)-\psi(s))^{\alpha-2} \psi^{\prime}(s) v_{1}(s) d s \\
+\frac{1}{\Gamma(\alpha)} \int_{a}^{t}(\psi(t)-\psi(s))^{\alpha-1} \psi^{\prime}(s) v_{1}(s) d s+\frac{1}{\Gamma(\alpha)} \int_{t}^{b}(\psi(s)-\psi(t))^{\alpha-1} \psi^{\prime}(s) v_{1}(s) d s
\end{gathered}
$$

By $\left(H_{6}\right)$, there exists $w \in F(t, \bar{u}(t))$ such that

$$
\left|v_{1}(t)-w(t)\right| \leq m(t)|u(t)-\bar{u}(t)|, t \in[a, b] .
$$

$U:[a, b] \rightarrow \mathcal{P}(R)$ is defined as

$$
U(t):=\left\{w \in R:\left|v_{1}(t)-w(t)\right| \leq m(t)|u(t)-\bar{u}(t)|\right\}
$$

The multivalued operator $U(t) \cap F(t, \bar{u}(t))$ is measurable, so there exits a measurable selection for $U(t) \cap F(t, \bar{u}(t))$. We denote this function as $v_{2}(t)$. For each $t \in[a, b]$, one has

$$
\left|v_{1}(t)-v_{2}(t)\right| \leq m(t)|u(t)-\bar{u}(t)|
$$


Then, we define for each $t \in[a, b]$,

$$
\begin{aligned}
& h_{2}(t)=-\frac{\psi(b)-\psi(a)}{2 \Gamma(\alpha-1)} \int_{a}^{b}(\psi(b)-\psi(t))^{\alpha-2} \psi^{\prime}(s) v_{2}(s) d s \\
& \quad+\frac{1}{\Gamma(\alpha)} \int_{a}^{t}(\psi(t)-\psi(s))^{\alpha-1} \psi^{\prime}(s) v_{2}(s) d s+\frac{1}{\Gamma(\alpha)} \int_{t}^{b}(\psi(s)-\psi(t))^{\alpha-1} \psi^{\prime}(s) v_{2}(s) d s,
\end{aligned}
$$

it follows that

$$
\begin{aligned}
\left|h_{1}(t)-h_{2}(t)\right| & \leq \frac{\psi(b)-\psi(a)}{2 \Gamma(\alpha-1)} \int_{a}^{b}(\psi(b)-\psi(t))^{\alpha-2} \psi^{\prime}(s)\left|v_{1}(s)-v_{2}(s)\right| d s \\
& +\frac{1}{\Gamma(\alpha)} \int_{a}^{t}(\psi(t)-\psi(s))^{\alpha-1} \psi^{\prime}(s)\left|v_{1}(s)-v_{2}(s)\right| d s \\
& +\frac{1}{\Gamma(\alpha)} \int_{t}^{b}(\psi(s)-\psi(t))^{\alpha-1} \psi^{\prime}(s)\left|v_{1}(s)-v_{2}(s)\right| d s \\
& \leq \frac{\psi(b)-\psi(a)}{2 \Gamma(\alpha-1)}\|u-\bar{u}\| \int_{a}^{b}(\psi(b)-\psi(t))^{\alpha-2} \psi^{\prime}(s) m(s) d s \\
& +\frac{1}{\Gamma(\alpha)}\|u-\bar{u}\| \int_{a}^{t}(\psi(t)-\psi(s))^{\alpha-1} \psi^{\prime}(s) m(s) d s \\
& +\frac{1}{\Gamma(\alpha)}\|u-\bar{u}\| \int_{t}^{b}(\psi(s)-\psi(t))^{\alpha-1} \psi^{\prime}(s) m(s) d s \\
& \leq\left[\frac{\psi(b)-\psi(a)}{2 \Gamma(\alpha-1)} \int_{a}^{b}(\psi(b)-\psi(t))^{\alpha-2} \psi^{\prime}(s) m(s) d s\right. \\
& \left.+\frac{2}{\Gamma(\alpha)}(\psi(b)-\psi(a))^{\alpha-1} \int_{a}^{b} \psi^{\prime}(s) m(s) d s\right]\|u-\bar{u}\| . \\
& =\gamma\|u-\bar{u}\| .
\end{aligned}
$$

Therefore,

$$
\left\|h_{1}-h_{2}\right\| \leq \gamma\|u-\bar{u}\| .
$$

Interchanging $u$ and $\bar{u}$ yields

$$
H_{d}(F(t, \bar{u}), F(t, u)) \leq \gamma\|u-\bar{u}\| .
$$

Thus, $T$ is a contraction by $\gamma<1$. Since Lemma 2, we conclude that $T$ admits a fixed point which is a solution to problem (4).

The third theorem is about the lower semicontinuous case.

Theorem 3. Assume that $\left(H_{1}\right)-\left(H_{4}\right)$ hold, if $\left(H_{7}\right)$ is also satisfied, then the anti-periodic boundary problem (4) has at least one solution on $[a, b]$.

Proof. It is clear that $F$ is of 1.s.c. type as condition $\left(H_{7}\right)$ is satisfied. By means of Lemma 4 , there exists a continuous function $f: C(J, R) \rightarrow L^{1}(J, R)$ such that $f(u) \in \mathcal{F}(u)$ for all $u \in C(J, R)$.

Next, we shall consider the following problem

$$
\left\{\begin{array}{l}
\left(\begin{array}{l}
R C \\
\psi(a)
\end{array} D_{\psi(b)}^{\alpha} u\right)(x)=f(u(x)), \quad \psi(a)<x<\psi(b), \\
u(\psi(a))+u(\psi(b))=0, \quad u^{\prime}(\psi(a))+u^{\prime}(\psi(b))=0,
\end{array}\right.
$$


Note that if $u \in C^{2}([a, b], R)$ is a solution to (42), then $u$ is a solution to the problem (4). we define the operator $\overline{\mathcal{T}}$ as

$$
\begin{aligned}
\overline{\mathcal{T}} u(x)= & -\frac{\psi(b)-\psi(a)}{2 \Gamma(\alpha-1)} \int_{a}^{b}(\psi(b)-\psi(s))^{\alpha-2} \psi^{\prime}(s) f(u(s)) d s \\
& +\frac{1}{\Gamma(\alpha)} \int_{a}^{t}(\psi(t)-\psi(s))^{\alpha-1} \psi^{\prime}(s) f(u(s)) d s+\frac{1}{\Gamma(\alpha)} \int_{t}^{b}(\psi(s)-\psi(t))^{\alpha-1} \psi^{\prime}(s) f(u(s)) d s .
\end{aligned}
$$

We transform the problem (42) into a fixed point problem. Obviously, the operator $\overline{\mathcal{T}}$ is continuous and completely continuous. As the remainder of the proof is similar to that of Theorem 1 , we omit it here.

Remark 2. If we take $F(x, u)=\{f(x, u)\}$, where $f:[a, b] \times R \rightarrow R$ is a given continuous function, then the problem (4) corresponds to the single-valued problem (1).

Remark 3. If we take $a=0, b=T, \psi(x)=x, F(x, u)=\{g(x, u)\}$, where $g:[0, T] \times R \rightarrow R$ is a given continuous function, then the problem (4) corresponds to the single-valued problem (2).

\section{Applications}

Example 1. Consider the fractional differential inclusion involving $\psi$-Riesz-Caputo derivative with anti-periodic boundary value conditions

$$
\left\{\begin{array}{l}
{ }_{-1}^{R C} D_{1}^{\frac{3}{2}, \psi} u(x) \in F(x, u(x)) \\
u(-1)+u(1)=0, u^{\prime}(-1)+u^{\prime}(1)=0
\end{array}\right.
$$

where $\psi(x)=\sinh (x), \quad-1 \leq x \leq 1 . \alpha=\frac{3}{2}$. Observe that $\psi \in C^{2}([-1,1]), \psi^{\prime}(x)=\cosh (x)>0$, $-1 \leq x \leq 1$. Moreover, we have

$$
\psi^{\prime}(-1)=\cosh (-1)=\cosh (1)=\psi^{\prime}(1),
$$

which implies condition $\left(H_{3}\right)-\left(H_{4}\right)$ hold.

$$
x \rightarrow F(x, u(x)):=\left[\frac{|u|^{5}}{|u|^{5}+3}+x^{2}+1, \frac{|u|}{|u|+1}+x^{3}+1\right], u \in R,
$$

and

$$
\|F(x, u)\|:=\sup |v|: v \in F(x, u) \leq 3:=p(x) q(\|u\|) u \in R .
$$

Obviously, condition $\left(H_{1}\right)$ is satisfied. And $p(x)=1, q(\|u\|)=3$, we can find a positive constant $M$ such that

$$
\begin{gathered}
M\left[3\left(\frac{|\sinh (1)-\sinh (-1)|}{2 \Gamma(1 / 2)} \int_{-1}^{1}(\sinh (1)-\sinh (s))^{-1 / 2} \cosh (s)\right) d s\right. \\
\left.\left.+\frac{2(\sinh (1)-\sinh (-1))^{1 / 2}}{\Gamma(3 / 2)} \int_{-1}^{1} \cosh (s) d s\right)\right]^{-1}>1,
\end{gathered}
$$

that is, $M>30.486$. All the conditions in Theorem 1 are satisfied. Therefore, the fractional differential inclusion with anti-periodic boundary value conditions (44) has at least one solution.

Example 2. Consider the fractional differential inclusion involving $\psi$-Riesz-Caputo derivative with anti-periodic boundary value conditions

$$
\left\{\begin{array}{l}
{ }_{-1}^{R C} D_{1}^{\frac{6}{5}, \psi} u(x) \in F(x, u(x)) \\
u(-1)+u(1)=0, u^{\prime}(-1)+u^{\prime}(1)=0
\end{array}\right.
$$


where $\psi(x)=\sin (x), \quad-1 \leq x \leq 1 . \alpha=\frac{6}{5}$. Observe that $\psi \in C^{2}([-1,1]), \psi^{\prime}(x)=\cos (x)>0$, $-1 \leq x \leq 1$. Moreover, we have

$$
\psi^{\prime}(-1)=\cos (-1)=\cos (1)=\psi^{\prime}(1),
$$

which implies condition $\left(H_{3}\right)-\left(H_{4}\right)$ hold.

$$
x \rightarrow F(x, u(x)):=\left[0, \frac{|x|}{3} \frac{|u|}{|u|+1}\right], u \in R,
$$

and

$$
d_{H}(F(t, u), F(t, \bar{u})) \leq \frac{|t|}{3}|u-\bar{u}|, u \in R
$$

we can find out that

$$
\begin{aligned}
\gamma & =\frac{\psi(1)-\psi(-1)}{2 \Gamma\left(\frac{1}{5}\right)} \int_{-1}^{1}(\psi(1)-\psi(s))^{-\frac{4}{5}} \psi^{\prime}(s) \frac{|s|}{3} d s+\frac{2}{\Gamma\left(\frac{1}{5}\right)}(\psi(1)-\psi(-1))^{\frac{1}{5}} \int_{-1}^{1} \psi^{\prime}(s) \frac{|s|}{3} d s \\
& =\frac{\sin (1)-\sin (-1)}{2 \Gamma\left(\frac{1}{5}\right)} \int_{-1}^{1}(\sin (1)-\sin (s))^{-\frac{4}{5}} \cos (s) \frac{|s|}{3} d s \\
& +\frac{2}{\Gamma\left(\frac{6}{5}\right)}(\sin (1)-\sin (-1))^{\frac{1}{5}} \int_{-1}^{1} \cos (s) \frac{|s|}{3} d s \\
& <0.967
\end{aligned}
$$

that is, $\gamma<1$. All the conditions in Theorem 2 are satisfied. Therefore, the fractional differential inclusion with anti-periodic boundary value conditions (45) has at least one solution.

\section{Conclusions}

Riesz derivative, which is different from one-sided fractional derivative, as the Caputo or Riemann-Liouville derivative, is a two-sided fractional operator. It is of great use due to its reflecting both the past and the future memory effects. We study the existence of solutions for a class of anti-periodic fractional differential inclusions with $\psi$-Riesz-Caputo fractional derivative in this paper. Firstly, combining $\psi$-Caputo derivative with Riesz-Caputo derivative, we give a new definition of $\psi$-Riesz-Caputo fractional derivative of order $\alpha$. Then, in virtue of fixed-point theorems for multi-valued maps, some sufficient conditions for the existence of solutions to the fractional differential inclusions are presented. Last but not least, we present two examples to illustrate our main results.

Author Contributions: Both authors contributed equally and significantly in writing this paper. Both authors read and approved the final manuscript.

Funding: This work is supported by the Natural Science Foundation of China (11571136).

Acknowledgments: The authors thanks anonymous referees for their remarkable comments, suggestion, and ideas that help to improve this paper.

Conflicts of Interest: The authors declare that they have no competing interests.

\section{References}

1. Ahmad, B.; Otero-Espinar, V. Existence of solutions for fractional differential inclusions with antiperiodic boundary conditions. Bound. Value Probl. 2009, 2009, 625347. [CrossRef]

2. Ahmad, B.; Ntouyas, S.K.; Zhou, Y.; Alsaedi, A. A Study of Fractional Differential Equations and Inclusions with Nonlocal Erdelyi-Kober Type Integral Boundary Conditions. Bull. Iran. Math. Soc. 2018, 44, 1315-1328. [CrossRef] 
3. Almeida, R. A Caputo fractional derivative of a function with respect to another function. Commun. Nonlinear Sci. Numer. Simula 2017, 44, 460-481. [CrossRef]

4. Almeida, R.; Malinowska, A.B.; Monteiro, M.T.T. Fractional differential equations with a Caputo derivative with respect to a kernel function and their applications. Math. Meth. Appl. Sci. 2018, 41, 336-352. [CrossRef]

5. Almeida, R.; Malinowska, A.B.; Odzijewicz, T. On systems of fractional differential equations with the $\psi$-Caputo derivative and their applications. Math. Meth. Appl. Sci. 2019. [CrossRef]

6. Kilbas, A.A.; Srivastava, H.M.; Trujillo, J.J. Theory and Applications of Fractional Differential Equations; North-Holl and Mathematics Studies: Elsevier: Amsterdam, The Netherlands, 2006.

7. Podlubny, I. Fractional Differential Equations; Academic Press: San Diego, CA, USA, 1999.

8. Wang, J.R.; Ibrahim, A.G.; O'Regan, D. Topological structure of the solution set for fractional non-instantaneous impulsive evolution inclusions. J. Fixed Point Theory Appl. 2018, 20, 59. [CrossRef]

9. Ahmad, B.; Ntouyas, S.K.; Alsaedi, A. On fractional differential inclusions with anti-periodic type integral boundary conditions. Bound. Value Probl. 2013, 2013, 82. [CrossRef]

10. Chen, F.L.; Chen, A.P.; Wu, X. Anti-periodic boundary value problems with Riesz-Caputo derivative. Adv. Differ. Equ. 2019, 2019, 119. [CrossRef]

11. Ahmad, B.; Ntouyas, S.K. On Hadmard fractional integro-differential boundary value problems. J. Appl. Math. Camput. 2015, 47, 119-131. [CrossRef]

12. Baghani, $\mathrm{H}$. Existence and uniqueness of solutions to fractional Langevin equations involving two fractional orders. J. Fixed Point Theory Appl. 2013, 20, 63. [CrossRef]

13. Baleanu, D.; Mousalou, A.; Rezapour, S. A new method for investigating approximate solutions of some fractional integro-differential equations involving the Caputo-Fabrizio derivative. Adv. Differ. Equ. 2017, 2017, 51. [CrossRef]

14. da C. Sousa, J.V.; Rodrigues, F.G.; de Oliveira, E.C. Stability of the fractional Volterra integro-differential equation by means of $\psi$-Hilfer operator. Math. Meth. Appl. Sci. 2019, 42, 3033-3043.

15. Vivek, D.; Kanagarajan, K.; Elsayed, E.M. Some existence and stability results for Hilfer-fractional implicit differential equations with nonlocal conditions. Mediterr. J. Math. 2018, 15, 15. [CrossRef]

16. Samet, B.; Aydi, H. Lyapunov-type inequalities for an anti-periodic fractional boundary value problem involving $\psi$-Caputo fractional derivative. J. Inequal. Appl. 2018, 2018, 286. [CrossRef] [PubMed]

17. Wu, G.; Baleanu, D.; Deng, Z.; Zeng, S. Lattice fractional diffusion equation in terms of a Riesz-Caputo difference. Physica A 2015, 438, 335-339. [CrossRef]

18. Yang, Q.; Liu, F.; Turner, I. Numerical methods for fractional partial differential equations with Riesz space fractional derivatives. Appl. Math. Model. 2013, 34, 200-218. [CrossRef]

19. Agrawal, O.P. Fractional variational calculus in terms of Riesz fractional derivatives. J. Phys. 2007, 40, 6287-6303. [CrossRef]

20. Chen, F.L.; Baleanu, D.; Wu, G.C. Existence results of fractional differential equations with Riesz-Caputo derivative. Eur. Phys. J. Spec. Top. 2017, 226, 3411-3425. [CrossRef]

21. Lakshmikantham, V.; Vatsala, A.S. Basic theory of fractional differential equations. Nonlinear Anal. 2008, 69, 2677-2682. [CrossRef]

22. Awadalla, M.; Yameni, Y.Y. Modeling exponential growth and exponential decay real phenomena by $\psi$-Caputo fractional derivative. J. Adv. Math. Comput. Sci. 2018, 28, 1-13. [CrossRef]

23. Luo, D.F.; Luo, Z.G. Existence and finite-time stability of solutions for a class of nonlinear fractional differential equations with time-varying delays and non-instantaneous impulses. Adv. Dffer. Equ. 2019, 2019, 155. [CrossRef]

24. Bohnenblust, H.F.; Karlin, S. On a theorem of Ville. In Contributions to the Theory of Games, Vol. I; Annals of Mathematics Studies; Princeton University Press: Princeton, NJ, USA, 1950; Volume 24, pp. 155-160.

25. Bressan, A.; Colombo, G. Extensions and selections of maps with decomposable values. Studia Math. 1988, 90, 69-86. [CrossRef]

26. Deimling, K. Multivalued Differential Equations; De Gruyter: Berlin, Germany, 1992.

27. Granas, A.; Dugundji, J. Fixed Point Theory; Springer: New York, NY, USA, 2005.

28. Papageorgiou; Shouchuan, H.; Nikolaos, S. Handbook of Multivalued Analysis Theory I; Kluwer: Dordrecht, The Netherlands, 1997. 
29. Covitz, H.; Nadler, S.B., Jr. Multivalued contraction mappings in generalized metric spaces. Israel J. Math. 1970, 8, 5-11. [CrossRef]

30. Lasota, A.; Opial, Z. An application of the Kakutani-Ky Fan theorem in the theory of ordinary differential equations. Bull. Acad. Pol. Sci. Ser. Sci. Math. Astronom. Phys. 1965, 13, 781-786.

(C) 2019 by the authors. Licensee MDPI, Basel, Switzerland. This article is an open access article distributed under the terms and conditions of the Creative Commons Attribution (CC BY) license (http://creativecommons.org/licenses/by/4.0/). 